CAHIERS DE

NARRATOLOGIE

\section{Cahiers de Narratologie}

Analyse et théorie narratives

$28 \mid 2015$

Le récit comme acte cognitif

\title{
Un regard esthétique sur la segmentation des films*
}

\section{Márcia Aurélio Baldissera}

\section{(2) OpenEdition \\ Journals}

Electronic version

URL: https://journals.openedition.org/narratologie/7247

DOI: $10.4000 /$ narratologie. 7247

ISSN: 1765-307X

Publisher

LIRCES

Electronic reference

Márcia Aurélio Baldissera, "Un regard esthétique sur la segmentation des films*", Cahiers de Narratologie [Online], 28 | 2015, Online since 29 October 2015, connection on 11 June 2021. URL: http://journals.openedition.org/narratologie/7247 ; DOI: https://doi.org/10.4000/narratologie.7247

This text was automatically generated on 11 June 2021.

Article L.111-1 du Code de la propriété intellectuelle. 


\title{
Un regard esthétique sur la segmentation des films*
}

\author{
Márcia Aurélio Baldissera
}

\section{AUTHOR'S NOTE}

*Je remercie Anne-Marie Havard (EHESS) pour sa lecture et ses suggestions au niveau linguistique.

1 La segmentation des événements est l'un des mécanismes cognitifs qui nous guident dans la compréhension du monde réel et du monde représenté, par la perception de la structure de ces flux d'événements. Le film est un flux fait d'événements - les plans visuels et les sons - dans un agencement en succession et simultanéité - la séquence audiovisuelle. Parce que la durée et l'ordre des sons et des plans visuels sont, de fait, fort variés d'un film à l'autre, ces structurations diverses impliquent des différences cognitives lors de leur réception spectatorielle. Cette question ne semble pas être bien comprise par les auteurs qui réalisent des études empiriques sur le cinéma. En effet, selon la plupart des expérimentations et des théories sur lesquelles ces expérimentations s'appuient, il existe un unique mode de faire et de comprendre le cinéma. Plus important, il n'existe guère de recherches comparatives qui essaient de tenir compte de la diversité de la production et de la réception de films dans le monde. C'est pourquoi, dans une perspective esthétique, nous chercherons ici des différences dans le traitement cognitif des films par les spectateurs, processus qui tient à la structure des films eux-mêmes, en essayant d'éclaircir le rôle de la segmentation dans l'expérience cinématographique à l'aide de travaux expérimentaux. Pour cela, nous introduirons brièvement les questions soulevées par la recherche sur la segmentation des films, à savoir le type d'attente, le type d'attention, le rapport à la mémoire et à la compréhension. Nous décrirons ensuite les différences dans le traitement attentionnel pour tâcher de démontrer le fonctionnement de la segmentation au niveau cérébral et ses implications cognitives. Cela posera la question des conditions phénoménologiques de ces processus cinématographiques, c'est-à-dire du rythme propre du film, du rythme 
de sa réception et du rythme de son traitement cognitif. Ainsi, nous tenterons de montrer le rôle de la segmentation en fonction des structures formelles-techniques des films.

\section{La segmentation des films : point sur les recherches en cours}

2 Ce que nous percevons, c'est de la persistance et du changement à travers un flux d'événements, c'est-à-dire le flux qui présente une situation réelle ou un objet technique : le flux perceptif d'un film, d'un texte, d'une musique, d'une bande dessinée, d'un jeu vidéo ${ }^{1}$. La perception est ainsi soutenue par une structure, à savoir la situation initiale d'un événement, son développement et son état final; et ainsi de suite pour le flux d'événements qui se déroule. La segmentation, quant à elle, est la division d'un flux en unités de signification dans l'ordre séquentiel présenté par l'objet perceptif. À travers ce processus, nous sommes amenés à créer des attentes, qui sont gérées grâce à la situation présente et à nos connaissances. De cette façon, dans une perspective écologique, qui concerne les rapports entre les êtres vivants et leur environnement, la segmentation collabore au traitement de la mémoire et à la planification des actions. De plus, dans l'activité de réception des récits, ces unités de signification forment un modèle situationnel, à savoir un modèle cognitif multidimensionnel qui représente la situation donnée et qui nous conduit à la compréhension du récit. Ce modèle géré par la mémoire de travail s'applique à l'objet de l'expérience courante en utilisant nos schémas cognitifs, qui sont les structures représentant nos connaissances générales acquises, qu'elles soient perceptives, pratiques, procédurales ou propositionnelles. Puisque les schémas représentent des expériences prototypiques, ils nous conduisent à former des attentes sur la manière dont ces flux factuels ou fictifs vont procéder. Pour la même raison, ce sont aussi les schémas qui nous aident à combler le manque d'information que l'on rencontre généralement dans les récits. Ainsi, chaque expérience peut renforcer nos croyances, actualiser ou adapter nos connaissances acquises, et/ou nous conduire à un apprentissage.

3 Afin de mieux comprendre le rôle de la segmentation dans notre compréhension du cinéma, nous analyserons ci-après les expérimentations de l'équipe de Jeffrey Zacks sur les films Moonraker et Le Ballon Rouge ${ }^{2}$; ensuite, nous discuterons les recherches d'Uri Hasson et de son équipe sur deux films de Charlie Chaplin; enfin, nous observerons celles de Siina Pamilo et de ses collègues sur le film At Land de Maya Deren (1944).

4 En travaillant sur des situations réelles, des textes et des films, Zacks ${ }^{3}$ a démontré que les mécanismes neuronaux qui soutiennent la segmentation de ces flux caractérisent un processus ascendant, à savoir préconscient et préattentionnel. Dans une des premières expérimentations ${ }^{4}$, les participants ont vu des situations triviales pendant la première scanographie du cerveau: une personne en train de faire le lit, de faire la vaisselle, de fertiliser une plante et de repasser une chemise, dans des enregistrements de quelques minutes d'un plan fixe et unique. Dans la deuxième vision, ils ont réalisé la tâche d'appuyer sur un bouton pour identifier les unités actancielles qui leur ont semblé " naturelles et significatives ». Ainsi, les participants ont identifié des frontières événementielles (boundaries), qui se sont avérées hautement corrélées entre les participants. Elles ont été identifiées lors d'un changement dans la situation représentée, un changement dû principalement à la dimension temporelle et au 
mouvement. Par conséquent, dans le cas des expérimentations avec des morceaux de films, chaque fois qu'une séquence actancielle s'accomplit, les participants détectent cette frontière qui mène à l'actualisation du modèle situationnel courant et à la formation d'attentes sur le procédé des actions. Cela démontre le rôle de la segmentation dans la planification des actions, qu'elles aient pour agent le sujet observant ou le sujet observé. Il faut remarquer que ces processus de segmentation, actualisation $\mathrm{du}$ modèle situationnel et formation d'attentes sont entièrement ascendants ; ces processus font partie de notre manière écologique d'interagir avec le monde.

5 Étant donné que ces frontières sont hautement corrélées entre les participants et qu'elles ont en outre une forte correspondance avec les coupes qui représentent la division en séquences spatio-temporelles du film lui-même, Zacks en déduit que le montage cinématographique joue un rôle primordial dans la segmentation des films. Plus exactement, il défend l'idée selon laquelle le style du montage analytique continu, dit " classique ${ }^{5}$ ", facilite la compréhension des spectateurs parce que leurs attentes sont satisfaites ${ }^{6}$. Cette conclusion se rapporte à l'expérimentation menée sur le film Moonraker (Gilbert, 1979), de la série de James Bond. Il faut remarquer que les attentes auxquelles Zacks fait référence sont des attentes de bas niveau ou automatiques, à savoir des attentes sur les actions des personnages dans un ordre causal et chronologique. Cependant, il existe des attentes de plus haut niveau par rapport au monde diégétique, telles celles relatives à la relation entre les personnages, à leurs motivations, croyances et émotions ; d'autres attentes peuvent arriver également d'un point de vue moins objectif, qui concerne la structure du récit et/ou des états mentaux plus implicites des personnages, ce qui peut entraîner un traitement de plus haut niveau relatif aux intentions des auteurs du film et/ou aux états mentaux du spectateur lui-même.

6 La formation d'attentes plus subjectives pourrait être ainsi vérifiée dans le film Le Ballon Rouge (Lamorisse, 19567), cité par Zacks ${ }^{8}$ pour soutenir sa proposition sur l'efficacité du montage classique dans la compréhension des spectateurs. Bien que la corrélation de la segmentation entre les participants ait coïncidé avec l'accomplissement des séquences actancielles, Le Ballon Rouge n'est pas un film de style classique : il n'est pas découpé et monté selon une stratégie analytique et continue pour mieux représenter un but; mais tout autrement, il est fait de plans d'une longue durée et sans aucun souci d'illusion de continuité entre eux, cela pour donner une sensation plus « réaliste » au récit ${ }^{9}$. La seule différence mentionnée ailleurs par Zacks ${ }^{10}$, entre ce film et les films classiques, est la faible activation de la sub-région du cortex visuel responsable de la détection du mouvement, appelée simplement MT+, qui selon l'auteur peut refléter la singularité des événements du film français : «Le Ballon Rouge contient de nombreux longs plans d'accompagnement (tracking shots) qui suivent le personnage dans l'action de courir (running), plans qui peuvent rendre l'information de bas niveau sur le mouvement moins prévisible (predictive ${ }^{11}$ ) ». Cette question est d'une importance fondamentale, parce qu'elle démontre que nous détectons la télicité ou l'atélicité des actions des autres sujets (réels ou fictifs) de manière également préattentionnelle, ce qui mène à la formation d'attentes sur l'accomplissement du mouvement qui sont, elles aussi, de bas niveau. Mais ces attentes ont des degrés différents dans leur satisfaction, en fonction de la télicité ou de l'atélicité des actions, ce qui peut conduire à des traitements cognitifs différents. Malgré cette mention, Zacks ne développe pas les implications de ces différences formelles, à savoir le rythme, dans le traitement cognitif 
des films. Cela est dû en partie à sa préoccupation d'exposer une théorie de la segmentation justifiée par la corrélation entre les sujets et entre les divers supports sémiotiques. Pourtant, ci-dessous, nous décrirons le fonctionnement de nos mécanismes attentionnels pour montrer les différences cognitives en fonction de la satisfaction ou de la non-satisfaction de nos attentes; ensuite, nous reviendrons sur la question du rythme.

\section{Les inflexions attentionnelles et leurs implications cognitives lors de la segmentation}

7 D'autres travaux expérimentaux sur le système attentionnel confirment que la segmentation des événements relève d'un processus ascendant ${ }^{12}$. D'après les recherches sur la perception ${ }^{13}$, le réseau ventral est connu comme réseau sémantique. De cette façon, dans la perspective attentionnelle, le réseau ventral est responsable de la sélection et de la surveillance du système dans son interaction avec le monde externe : non seulement en distinguant les stimuli pertinents par leur saillance et par leur pertinence comportementale, émotionnelle et sémantique ; mais aussi en filtrant les stimuli qui ne sont pas pertinents, en maintenant ainsi le traitement focalisé en cours par le système attentionnel. Comme le montrent les expérimentations de Zacks, cité par Corbetta, Patel et Shulman, lors de la segmentation d'un événement, l'activité marquée du réseau ventral indique une frontière qui déclenche l'actualisation du modèle situationnel par le réseau dorsal. Le réseau dorsal est appelé réseau pragmatique, parce que sa fonction est relative au suivi (monitoring) des actions pour leur planification; donc, conjointement à la mémoire de travail, le réseau dorsal s'occupe de ces événements dans une séquence, ce qui entraîne des attentes sur la situation à venir. Ces attentes peuvent être satisfaites à des degrés et des vitesses différents, et c'est leur non-satisfaction qui peut envoyer des signaux de haut niveau au réseau ventral pour éveiller notre attention. Autrement dit, selon les travaux de Zacks sur la segmentation, il s'agit de la réorientation du système attentionnel pour l'actualisation automatique du modèle situationnel en cours qui nous amène à la compréhension du récit. Mais l'activation du réseau ventral peut aussi indiquer un processus complètement différent de la réorientation attentionnelle: une «transgression" effective des attentes peut conduire à des signaux de haut niveau, à l'éveil attentionnel qui soutient l'apprentissage et qui peut mener à la satisfaction affective. C'est-à-dire que l'attention est appelée à résoudre le manque de satisfaction de nos attentes. Ou mieux, l'attention est appelée à rechercher la cohérence entre les événements dans l'intervalle entre les actions, en s'appuyant sur la perception des détails de l'objet et sur les connaissances d'arrière plan du sujet.

8 Néanmoins, Zacks, remarquant que la plupart des participants à l'expérimentation sur le film Moonraker ont vu leurs attentes satisfaites quant à la réussite de Bond, continue à postuler l'« efficacité » cognitive du style classique. Ses conclusions portent sur trois points : la valorisation de la télicité des actions; la valorisation du traitement orienté par les stimuli visuels; et l'association de la compréhension et de la mémoire à ce type de processus cognitif. «La rupture intentionnelle des règles du montage continu peut être souhaitée à des fins artistiques, écrit-il, et elle peut ne pas perturber le traitement perceptif de bas niveau. Cependant, précise-t-il, il pourrait y avoir des coûts pour la compréhension et pour la mémoire dans la violation de ces conventions ${ }^{14}$ ». En fait, 
cette conclusion contredit les données des recherches actuelles sur l'attention et les recherches de Zacks lui-même en dehors du cadre cinématographique. Dans un autre article $^{15}$ il éclaircit, en effet, la relation entre attention, actualisation de la mémoire et échec de nos attentes: "lier l'attention - et principalement l'actualisation de la mémoire - aux échecs des attentes peut être hautement adaptatif. Si la non-satisfaction de nos attentes survient aux moments où un nouvel événement significatif a commencé, c'est alors exactement ce moment dont on devrait actualiser les modèles mentaux, en fonction de «ce qui se passe maintenant » [...] Ce mécanisme, poursuit-il, peut être très précieux pour la régulation immédiate du comportement, autant que pour orienter l'apprentissage sur notre environnement ${ }^{16} »$. En somme, il semble que les conclusions de Zacks révèlent une admission implicite que le traitement cognitif des films classiques - orienté par le dénouement de l'histoire racontée à travers un flux causal d'actions et de résultats continuellement représentés - requiert une attention orientée par un but externe (le but du récit du film lui-même), attention qui sera plus objective et pragmatique.

9 L'expérience cinématographique peut aussi être bien plus personnelle, dans le sens où elle peut avoir un point de vue plus subjectif et être orientée par les états mentaux internes du spectateur lui-même. Le réseau ventral peut, également, réorienter notre attention vers l'autre réseau attentionnel, le default-mode. Ce réseau s'active quand nous n'avons pas de but ou de tâche à accomplir, mais lorsque nous sommes focalisés sur un processus interne, tel que la mémoire de notre propre passé, l'imagination de nos propres états mentaux ou de ceux d'autrui, ou encore l'introspection. Par conséquent, le default-mode et le réseau dorsal se contraignent réciproquement, ce qui montre que l'attention est focalisée sur le traitement des processus internes, ou bien elle l'est sur le traitement des processus externes, qu'ils soient diégétiques ou environnementaux. Le réseau ventral semble interagir avec le réseau default-mode dans la transition entre une perspective interne (corporelle ou introspective) et une perspective externe, qu'elle soit environnementale, ou qu'il s'agit de la perspective d'autrui (réelle ou fictionnelle). Plus spécifiquement, la TPJ (jonction temporopariétale), région qui fait partie du réseau default-mode, est active pendant certains processus cognitifs de bas niveau tels que la réorientation de l'attention et le sens d'agentivité (sense of agency), et aussi pendant des processus de haut niveau tels que l'empathie et les tâches de mindreading ${ }^{17}$, à savoir des tâches dans lesquelles les participants attribuent des états mentaux à autrui (leurs motivations, croyances, émotions). Comme pour tous les processus de haut niveau - qui indiquent un effort attentionnel affectif et/ou sémantique - les temps de réaction comportementale, qui correspondent à la réponse neuronale, sont aussi plus longs quand on fait une expérience caractérisée comme empathique, par exemple. Autrement dit, plus on a du temps pour le traitement attentionnel d'une représentation mentale, plus cette représentation peut être enrichie par les détails de l'objet perceptif et par nos connaissances d'arrière plan.

10 Nous avons affaire, alors, à un processus dans lequel l'interaction et la dynamique entre les réseaux cérébraux indiquent, au niveau neuronal, s'il s'agit de notre rapport au monde externe ou au 'monde' interne. Autrement dit, l'interaction entre les réseaux attentionnels permet d'interpréter s'il s'agit d'une activité relevant plutôt d'une immersion totale ou partielle, c'est-à-dire que c'est l'activation marquée du defaultmode qui indique clairement une immersion partielle. Cette focalisation sur le traitement dans les régions du default-mode n'empêche pas, pour autant, le traitement 
dans les régions du réseau dorsal, et vice-versa, principalement lors de la réception d'un film, lorsqu'il faut que le monde diégétique et notre "monde " subjectif interagissent dans un déroulement temporel. C'est cette dynamique qui nous mène, à présent, à une question fondamentale dans le processus cognitif d'un film: sa phénoménologie.

\section{Rythme : les temps du traitement cognitif, les temps de réaction, les temps du film}

11 Uri Hasson ${ }^{18}$ a développé des expérimentations avec des extraits de films qui abordent la question de l'étendue temporelle nécessaire à la réponse des aires cérébrales, laquelle dépend $d u$ parcours et du temps disponible pour le traitement d'une représentation mentale. De la même façon qu'il existe une hiérarchie cérébrale par rapport au traitement des propriétés spatiales des événements, à savoir une intégration parallèle et consécutive de ces propriétés dans une progression temporelle, Hasson défend l'idée selon laquelle il y a aussi une hiérarchie par rapport au traitement des propriétés temporelles. C'est-à-dire que : i) les propriétés temporelles des événements sont traitées ii) dans une hiérarchie temporelle qui peut être affectée par ces mêmes propriétés temporelles. Ce qui est donc mesuré dans cette expérimentation, c'est la hiérarchie temporelle des régions responsables pour le traitement de l'ordre d'une séquence d'événements. L'hypothèse est que l'étendue de la réponse de chaque région cérébrale doit correspondre à son rôle fonctionnel. De fait, cette étude confirme que les stimuli perceptifs sont traités rapidement par les aires cérébrales de bas niveau, alors que plus le traitement des représentations mentales s'élève, plus ce traitement est lent.

Dans cette expérimentation, Hasson a utilisé deux extraits de films de Charlie Chaplin, The Adventurer (1917) et City Lights (1931 ${ }^{19}$ ), d'environ 3,39 minutes chacun. Ces extraits ont été ultérieurement divisés en leurs plans originaux, d'environ 6 secondes, et remontés selon trois rythmes différents pour varier leur degré de "cohérence temporelle", à savoir l'ordre chronologique de l'histoire. Cette procédure sert à comparer la fiabilité (reliability) du traitement conduit par un film qui a une structure cohérente et chronologique au traitement qui advient quand la structure temporelle du film est «perturbée ». Il faut, cependant, remarquer la différence entre cohérence et ordre chronologique: l'ordre chronologique repose, en effet, sur notre manière écologique d'interagir avec le monde externe, ce qui n'est pas obligatoirement le cas pour les récits. Ainsi, la cohérence est indépendante de l'ordre chronologique. Mais, dans le cas spécifique de cette expérimentation, les films sont utilisés comme exemples de situations 'réelles' ou 'écologiques' dans le fonctionnement de nos processus cognitifs.

13 Ainsi, chaque participant a vu 8 extraits filmiques différents deux fois, 4 extraits du film The Adventurer et 4 extraits du film City Lights, d'environ 3,39 minutes chacun, selon 4 périodes différentes:

14 i) deux extraits, chacun d'un film différent, représentaient la période écologique, à savoir celle respectant le rythme et l'ordre chronologique originaux des films ;

ii) deux extraits représentaient une période de réponse longue, c'est-à-dire qu'ils avaient conservé les premières 36 secondes de la structure originale du film, suivies par une succession de plans d'environ 6 secondes aléatoirement remontés ; 
iii) deux extraits représentaient une période intermédiaire, c'est-à-dire que les premières 12 secondes avaient été préservées, suivies d'un remontage aléatoire de plans de 6 secondes;

iv) deux extraits représentaient une période courte, dont chacun avait été remonté au hasard, selon la fréquence de 4 secondes pour chaque plan.

15 De cette façon, chaque période des extraits cinématographiques, défini par la durée de la séquence dans son ordre chronologique original, correspond à une étendue de réponse, à savoir 4,12 et 36 secondes. C'est-à-dire que, selon l'hypothèse de l'auteur, dans les régions cérébrales qui répondent prioritairement du traitement instantané des stimuli sensoriels, les réponses doivent être similaires lors des présentations répétées des différents extraits filmiques. En revanche, dans les régions dans lesquelles la réponse dépend de l'ordre chronologique de la séquence, la corrélation des présentations doit dépendre des périodes de chaque extrait filmique.

16 Les conclusions de l'étude de Hasson peuvent être distinguées en trois questions: l'ordre des événements ; la chronologie des événements ; et la durée des événements.

17 Du point de vue de l'ordre des événements, les résultats de la scanographie démontrent que la fiabilité des régions de bas niveau n'a pas été affectée par la structure temporelle des extraits filmiques, ce qui indique clairement que les groupes de neurones de ces régions ont une étendue de réponse courte, d'une seconde ou moins; pourtant, les régions de plus haut niveau sont affectées par le traitement cognitif des représentations mentales constituées par les extraits altérés. Autrement dit, d'après Hasson: «l'ordre d'un groupe identique d'événements dans une même étendue séquentielle peut avoir un fort effet dans les réponses des régions cérébrales qui accumulent de l'information dans le temps, mais un faible effet dans les régions d'étendue courte. Les étendues de réponse courte, observées dans le cortex visuel, soutiennent l'idée que ces régions cérébrales sont rendues optimales pour le traitement instantané des propriétés visuo-spatiales d'un stimulus. Toutefois, plusieurs processus cognitifs (par exemple la communication verbale, la segmentation des événements, le mindreading, etc.) requièrent une accumulation d'informations dans le temps ${ }^{20} »$. Les conclusions de Hasson soutiennent donc l'hypothèse selon laquelle l'ordre des événements exerce une influence déterminante dans la compréhension des spectateurs. Ainsi, en suivant l'auteur, on peut établir une hiérarchie cognitive temporelle: les régions sensorielles (la MT + incluse) ont une activité presque instantanée; ensuite, dans une étendue équivalente à 12 secondes, on trouve les régions qui semblent intégrer les informations dans la séquence d'événements, qui relèvent, par exemple, de la différence du traitement cognitif selon les styles de montage des plans cinématographiques; et dans l'étendue longue, de 36 secondes à la durée totale de l'extrait, on trouve les régions (incluse la TPJ) qui semblent nécessaires pour simuler les motivations, les croyances et les émotions des personnages; pour établir et actualiser le modèle situationnel ; et pour gérer les attentes. Certaines de ces régions avaient déjà été relevées par les expérimentations de Zacks lors de la segmentation des événements, ce qui confirme le parcours ascendant et préattentionnel de ce mécanisme. Hasson remarque, quant à lui, que ces résultats ne déterminent pas que chaque région cérébrale a une étendue de réponse fixe. De fait, ces étendues de réponse spécifiques, notamment 4,12 et 36 secondes, se rapportent aux durées déterminées des extraits filmiques de cette expérimentation. En outre, les données empiriques des neurosciences ont démontré la plasticité architecturale et 
temporelle de notre cerveau en fonction de l'apprentissage, c'est-à-dire que les temps de réponse sont plus rapides pour un sujet expert dans une activité spécifique.

18 Le deuxième point à considérer dans cette étude porte à la fois sur la chronologie de l'ordre des événements, et sur l'effort de compréhension de chaque participant: l'altération de la structure temporelle des extraits n'a pas affecté l'amplitude des réponses dans les régions cérébrales de l'étendue longue dans lesquelles la fiabilité des réponses a été «notablement » réduite. Pour la raison que non seulement l'ordre nonchronologique n'a pas empêché le traitement cognitif des extraits altérés, ce qui confirme le fait que la cohérence n'est pas liée à l'ordre chronologique du récit ; mais aussi que la corrélation ou fiabilité des réponses entre les sujets est dissociée de l'effort ou de l'amplitude du traitement cognitif de chaque spectateur. Autrement dit, le manque de corrélation entre les participants ne signifie pas qu'il n'existe pas de traitement attentionnel, puisque l'engagement attentionnel relève des processus cognitifs et des états mentaux (croyances, désirs, connaissances acquises) de chaque individu singulier, et non pas nécessairement de tout un groupe de personnes. De fait, Hasson a observé de grandes amplitudes de réponse dans les régions longues, parmi lesquelles on trouve la TPJ, pour toutes les versions des extraits filmiques. L'auteur poursuit: «nous interprétons cette forte réponse dans ces régions cérébrales comme indiquant un traitement incessant, visant sans doute à extraire des informations sémantiquement cohérentes de ces stimuli. En même temps, la basse fiabilité des réponses aux films remontés indique un échec à atteindre une séquence cohérente ${ }^{21} \ldots$ ». C'est-à-dire que chaque participant a cherché la cohérence des extraits filmiques indépendamment de leur ordre chronologique; mais, du fait que certains extraits ont été remontés au hasard, sans aucun souci de leur donner une cohérence, cet effort de compréhension a échoué. Cela démontre que notre attitude écologique face aux irrégularités est d'essayer de les comprendre, ce qui indique l'éveil attentionnel qui dépend d'emblée du temps dont nous disposons pour la satisfaction de cette recherche de cohérence entre les événements. Or, cette condition temporelle relève bien de la durée et de l'ordre des événements qui forment la situation représentée, parce que c'est cette extension temporelle propre au film qui conduit le traitement cognitif du film lui-même. Nous pouvons donc dire avec Jean-Marie Schaeffer que le style formel d'un objet sémiotique, c'est son style cognitif ${ }^{22}$.

19 Cela nous conduit à notre troisième et dernier point, celui de la durée des événements. En la matière, Hasson lui-même remarque l'importance de la durée dans notre compréhension des événements: "nous présumons, par conséquent, que chaque région cérébrale est accordée à un traitement cognitif dans une étendue de temps qui est écologiquement appropriée au contenu des représentations traitées. De cette façon, il devrait être difficile de traiter des représentations mentales à un rythme significativement plus rapide ou plus lent que son étendue écologiquement appropriée ${ }^{23}$ ». Cette conséquence cognitive, en fonction de la vitesse des stimuli, nous fait revenir à la question de la durée des plans cinématographiques. Ces événements contiennent en eux-mêmes plusieurs informations, telles que le point de vue, l'étendue spatiale et le mouvement du cadre lui-même ; les états et les actions des personnages ; des objets variés ; pour ne pas mentionner la dimensionnalité sonore et son rapport à l'image. Comme nous l'avons déjà indiqué, il y a une différence cognitive en fonction de la durée de l'action dans un plan. Si la détection de la télicité des actions dans le film Moonraker est rendue de façon appropriée par les durées de ses plans, les plans atéliques du film Le Ballon Rouge sont ainsi détectés par les actions du personnage qui 
sont remarquables par ses durées très étendues. En effet, si la durée d'une action est plus courte que son étendue écologiquement appropriée, nous percevons une action télique, comme le montrent les expérimentations de Rolf Zwaan ${ }^{24}$ : l'événement est instantané, c'est-à-dire que nous percevons seulement son état final, la réussite de l'action. Donc, si les films contemporains ont un rythme beaucoup plus rapide, comme c'est le cas dans la plupart des films de style classique ${ }^{25}$, ce rythme devrait aussi avoir une conséquence cognitive.

20 Une première étude montre, de fait, que l'évaluation des sujets sur la difficulté à suivre un récit (difficulty to follow) est définitivement élevée lorsqu'une séquence visuelle a un rythme au-dessous du seuil de 2 à 3 secondes par plan ${ }^{26}$. Parce que l'information sur les événements s'accumule sur une étendue de quelques secondes, Fairhall et ses collègues attribuent ce seuil non pas au contenu perceptif changeant des images cinématographiques elles-mêmes, mais à l'étendue temporelle nécessaire pour l'individuation consciente de tout contenu perceptif événementiel complexe, c'est-àdire d'objets mouvants en succession et en simultanéité dans un environnement. Ils suggèrent que ce mécanisme temporel inné a la fonction de donner des représentations mentales stables et cohérentes d'un monde toujours en changement. Comme les films ici discutés ont tous un rythme supérieur à 2-3 secondes par plan, nous proposons de poursuivre notre analyse sur la segmentation avec une étude sur le traitement cognitif d'un film qui ne suit pas, délibérément, un ordre chronologique.

21 Cette expérimentation de Pamilo et collègues, réalisée avec le film muet At Land de Maya Deren $^{27}$ (1944), a eu comme objectif d'éclaircir le rôle fonctionnel des réseaux cérébraux dans une haute dimensionnalité, c'est-à-dire d'identifier la hiérarchie et les sub-unités des réseaux cérébraux par le temps de parcours des représentations mentales, qui est le temps de réponse à ces représentations. At Land, film qui représente la poétique surréaliste et qui a un style très différent du style classique, a été choisi pour sa capacité immersive et pour son nombre élevé de représentations d'actions capables d'activer les régions responsables de notre perception sensorimotrice. De fait, l'activation marquée du default-mode a indiqué clairement une immersion attentionnelle partielle, alors que les régions sensorimotrices se sont moins activées que ce qu'espéraient les auteurs. L'expérimentation avec le film de Deren montre, en outre, le déroulement et la plasticité d'un traitement cognitif de plus haut niveau par rapport à l'expérimentation de Hasson; tandis que l'ensemble de ces deux études démontre que la corrélation des processus cognitifs entre les participants est plus stable dans les aires visuelles. Cette conclusion est pleinement cohérente, car la perception est un processus dont les stimuli ont un rôle causal ; même s'il existe des différences dans les degrés de corrélation entre les sujets, principalement en fonction de la focalisation de l'attention sur des stimuli différents dans un même plan, ou sur un processus interne du spectateur lui-même.

22 Les participants à cette expérimentation ont regardé le film At Land pendant ses 15 minutes de durée totale, sans aucune instruction. Quatre dimensionnalités de la fonctionnalité cérébrale ont été mesurées. Dans la dimensionnalité la plus basse, qui correspond à des temps de parcours plus rapides, la scanographie a présenté une activité marquée dans le réseau dorsal conjointement aux aires visuelles, et également dans le réseau default-mode. Au fur et à mesure que l'on atteignait une dimensionnalité plus haute et lente, les réseaux dorsal et default-mode se sont divisés en régions toujours plus spécifiques, et, comme nous l'avons déjà expliqué antérieurement, leur 
fonctionnalité s'est nettement distinguée. Mais ce qui est plus important, ce sont les interprétations de Pamilo et de ses collègues sur les inflexions attentionnelles : dès la première dimensionnalité, la plus rapide, et jusqu'à la deuxième, "les parties supérieures du réseau dorsal se sont détachées du réseau inférieur et des régions visuelles. La partie supérieure du réseau dorsal a paru fonctionner plus comme un réseau attentionnel descendant, sans connexion aux régions visuelles ${ }^{28}$ »; et dans la dimensionnalité la plus lente, poursuivent-ils, « le cortex préfrontal médian (MPFC) s'est divisé dans deux unités du default-mode, une couvrant la partie ventrale du MPFC, et l'autre la dorsale. Selon les études précédentes, le MPFC dorsal participe du traitement affectif et émotionnel, tandis que le MPFC ventral est actif lors du traitement attentionnel de haut niveau ${ }^{29}$ ». Donc, dans la dimensionnalité la plus haute et lente, le default-mode a couvert la partie dorsale d'un hémisphère de haut niveau, ce qui démontre aussi une hiérarchie entre ces réseaux lors d'un traitement subjectif et attentionnel. En outre, les auteurs remarquent que seule la partie ventrale du PCC (cortex cingulaire postérieur) a montré une connexion fonctionnelle avec d'autres parties du default-mode, tandis que ses autres parties ont été connectées au réseau dorsal. Le point intéressant, c'est que les régions citées, MPFC et PCC, font partie d'un groupe de régions qui comprennent la TPJ, et qui semblent responsables de notre cognition sociale. En fonction de cette caractéristique, certains auteurs commencent à défendre l'idée selon laquelle le default-mode serait notre « cerveau social ${ }^{30} »$. De plus, il semble beaucoup plus intéressant également de penser la fonctionnalité cérébrale par réseaux, car la division par aires ne correspond pas à une fonction unique, surtout dans une expérience complexe et étendue comme celle de la réception d'un film.

Ces conclusions sur l'expérimentation avec le film At Land montrent que c'est cette dynamique des inflexions de l'attention qui révèle notre interaction lors d'une expérience cinématographique. Cependant, il faut du temps, même si cela relève de l'ordre des millisecondes, pour changer l'orientation du focus de l'attention; et il en faut bien plus, de l'ordre des secondes, pour le traitement attentionnel d'une représentation mentale d'un stimulus événementiel complexe. Or, il existe une condition phénoménologique dans une expérience cinématographique : c'est le temps mis à disposition des spectateurs qui est contrôlé par le film lui-même, ou, pour être plus précis, par le ou les auteurs du film. C'est-à-dire que pour favoriser l'engagement attentionnel du spectateur, il faut parvenir à un équilibre entre le temps donné pour la perception du film et le temps nécessaire pour les processus cognitifs de compréhension de ce film. Ainsi, dans une perspective esthétique, l'intérêt est de savoir quelles sont les implications dans le traitement attentionnel plus subjectif des spectateurs par rapport aux différentes structures temporelles des films. Autrement dit, de quelle manière le montage cinématographique, qui définit la durée et l'ordre des plans, influence-t-il la compréhension des spectateurs, et peut-il engendrer ou non un apprentissage?

\section{Le montage cinématographique : l'objet de l'interaction spectatorielle}

24 Comment établir des différences cognitives entre Moonraker, Le Ballon Rouge et At Land, par exemple? Dans le premier temps de la segmentation, celui de la détection des unités sémantiques, nous devons considérer la télicité et la structure des événements 
qui ont une durée déterminée, ce qui mène à des attentes de bas niveau. Moonraker est fait d'actions téliques; Le Ballon Rouge présente plutôt des états et des actions atéliques ; et At Land a été lui aussi conçu par états et actions atéliques, bien que plus rapides que celles du Ballon Rouge. Le processus qui suit, c'est le traitement cognitif de ces unités événementielles dans une séquence, traitement de bas niveau qui repose sur l'ordre des événements et qui doit satisfaire ou non les attentes créées antérieurement. C'est-àdire que dans le cas de Moonraker, formé d'actions téliques présentées selon un ordre causal et chronologique, la séquence est traitée plutôt de manière préattentionnelle parce qu'elle est objective; tandis que l'attention semble s'orienter vers le monde diégétique, c'est-à-dire sur les relations et les états mentaux des personnages. En revanche, Le Ballon Rouge et At Land, formés surtout par des états et des actions atéliques, et selon un ordre non-causal, répétitif, discontinu, avec des intervalles marqués, etc., semblent demander une attention plus subjective aux spectateurs. Mais Le Ballon Rouge et At Land sont formellement bien différents : en fonction de l'atélicité et de la durée de ses plans, le film français semble suspendre les attentes des spectateurs, parce qu'il n'y a pas une fin assurée, et de cette manière, l'attention semble se déplacer du niveau de l'action au niveau des états mentaux plus implicites du personnage principal ; alors que les intervalles marqués du film surréaliste éveillent notre attention sur la structure extradiégétique, ou formelle, du récit, attention qui semble avoir tendance à se focaliser sur les états mentaux de la réalisatrice et de son protagoniste. Ces deux films, Le Ballon Rouge et At Land, semblent donc focaliser notre attention sur les régions du default-mode par des moyens effectivement divers, tandis que l'attention sur le film classique semble être orientée par le réseau dorsal. Malheureusement, nous n'avons pas de données à la première personne, à savoir des données relatives à l'évaluation consciente des films par chaque spectateur, qui seules nous permettraient de vérifier le possible apprentissage favorisé par ces expériences. Cependant, d'après les données empiriques des recherches sur les réseaux ventral/default-mode et dorsal, nous pouvons inférer des implications cognitives en relation à l'activité marquée dans l'un de ces réseaux, et à la suspension ou non des attentes et des croyances des spectateurs. C'est-à-dire que si l'activité marquée des régions ventrales et du defaultmode relèvent d'un traitement cognitif plus attentionnel, d'une immersion partielle, d'une perspective plus allocentrique, d'une expérience phénoménologiquement plus riche, nous pouvons supposer la possibilité de la suspension des attentes et des croyances des spectateurs, condition nécessaire à un apprentissage procédural subjectif-social, qui pourrait donner lieu aussi à une expérience esthétique. Par contre, l'activité marquée des régions du réseau dorsal indique un traitement plus automatique ou préattentionnel, une immersion davantage focalisée sur le monde diégétique, une perspective plus égocentrique et phénoménologiquement plus pauvre, et ainsi, nous pouvons supposer qu'elle est une condition plus adaptée au renforcement des croyances des spectateurs, ce qui pourrait donner lieu à un apprentissage perceptif ou propositionnel.

En conclusion, nous pouvons avancer que la segmentation des films opère déjà au niveau du plan comme action dans une durée et, ensuite, au niveau de l'ordre de ces plans. C'est à travers ce mécanisme que nous formons des attentes et que les conditions empiriques de leur satisfaction nous sont posées. Et c'est encore à travers ce processus que nous arrivons à un certain type de compréhension du récit, plus pragmatique ou plus subjectif. De cette manière, il nous semble que cette expérience cinématographique dépend des structures temporelles - durée et ordre- qui 
soutiennent tant notre compréhension du monde que les films eux-mêmes. Parce que nos processus cognitifs se déroulent dans une temporalité qui leur est propre, et selon la temporalité de l'objet perceptif lui-même, le cinéma est, effectivement, doté d'une puissance cognitive remarquable. Étant donné que les implications de cette puissance cognitive sont fort différentes, à savoir qu'elle peut engendrer aussi bien un traitement presque automatique de renforcement des croyances qu'un traitement qui rend possible une expérience esthétique, il conviendrait sans doute de donner plus d'attention à cette relation cognitive spécifique, qui nous mène à comprendre le monde réel et à agir en lui et, peut-être, sur lui.

\section{BIBLIOGRAPHY}

Bazin, André (1958). Montage interdit. In Qu'est-ce que le cinéma ?, 20é éd., Paris : Cerf, pp. 49-61.

Bordwell, David (2006). The Way Hollywood Tells it. Berkeley et Los Angeles: University of California Press.

Corbetta, Maurizio; Patel, Gaurav et Shulman, Gordon (2008). The Reorienting System of the Human Brain: From Environment to Theory of Mind. Neuron, 58, pp. 306-324.

Cutting, James et al. (2011). Quicker, Faster, Darker : Changes in Hollywood film over 75 years. $i$ Perception, 2, pp. 569-576.

Decety, Jean et Lamm, Clauss (2007). The Role of the Right Temporoparietal Junction in Social Interaction: How Low-Level Computational Processes Contribute to Meta-Cognition.

Neuroscientist, 13 (6), pp. 580-593.

Fairhall, Scott et al. (2014). Temporal Integration Windows for Naturalistic Visual Sequences. PLoS ONE, 9 (7), URL: http://journals.plos.org/plosone/article?id=10.1371/journal.pone.0102248

Hasson, Uri et al. (2008). A Hierarchy of Temporal Receptive Windows in Human Cortex. Journal of Neuroscience, 28 (10), pp. 2539-2550.

Hasson, Uri et al. (2009). Reliability of Cortical Activity During Natural Stimulation. Trends in Cognitive Sciences, 14 (1), pp. 40-48.

Jacob, Pierre et Jeannerod, Marc (2003). Ways of Seeing. The Scope and Limits of Visual Cognition. New York: Oxford University Press.

Magliano, Joseph et Zacks, Jeffrey (2011). The Impact of Continuity Editing in Narrative Film on Event Segmentation. Cognitive Science, 35 (8), pp. 1489-1517.

Mars, Rogier et al. (2012). On the Relationship between the « Default Mode Network » and the « Social Brain ». Frontiers in Human Neuroscience, 6, pp. 1-9, URL : http://journal.frontiersin.org/ Journal/10.3389/fnhum.2012.00189/full

Pamilo, Siina et al. (2012). Functional Subdivision of Group-ICA Results of fMRI Data Collected during Cinema Viewing. PLoS ONE, 7 (7), URL: http://www.plosone.org/article/ info\%3Adoi\%2F10.1371\%2Fjournal.pone.0042000 
Schaeffer, Jean-Marie (2010). Esthétique et styles cognitifs : le cas de la poésie. Critique, $\mathrm{n}^{\circ}$ 752-753, pp. 59-70.

Zacks, Jeffrey et al. (2001). Human Brain Activity Time-Locked to Perceptual Event Boundaries. Nature Neuroscience, 4 (6), pp. 651-655.

Zacks, Jeffrey et al. (2010). The Brain's Cutting-Room Floor : Segmentation of Narrative Cinema. Frontiers in Human Neuroscience, 4, URL : http://journal.frontiersin.org/Journal/10.3389/fnhum. 2010.00168/full

Zacks, Jeffrey et al. (2011). Prediction Error Associated with the Perceptual Segmentation of Naturalistic Events. Journal of Cognitive Neuroscience, 23 (12), pp. 4057-4066.

Zacks, Jeffrey et Magliano, Joseph (2011). Film, Narrative, and Cognitive Neuroscience. In Melcher et Bacci (dir.), Art \& the Senses, New York: Oxford University Press, pp. 435-454.

Zwaan, Rolf (2008). Time in Language, Situation Models, and Mental Simulations. Language Learning 58 (1), pp. 13-26.

\section{NOTES}

1. Ce sont les objets des expérimentations courantes sur la segmentation. Cependant, il existe d'autres artefacts qui relèvent également d'un processus de segmentation lors de leur réception, par exemple la littérature orale, le théâtre, la danse.

2. Moonraker (Lewis Gilbert, 1979); Le Ballon Rouge (Albert Lamorisse, 1956).

3. Zacks et al. (2010). The brain's cutting-room floor: segmentation of narrative cinema. Frontiers in Human Neuroscience, 4, URL: http://journal.frontiersin.org/Journal/10.3389/fnhum.2010.00168/ full

4. Zacks et al. (2001). Human brain activity time-locked to perceptual event boundaries. Nature Neuroscience, 4 (6), pp. 651-655.

5. Par montage analytique continu, ou classique, on désigne le cinéma de siège hollywoodien qui caractérise les films mainstream. En termes brefs, la technique de tournage et de montage a comme norme la construction d'un flux perceptif de continuité spatio-temporelle des plans visuels et des événements sonores. Le récit suit, ainsi, une structuration des représentations audiovisuelles selon une logique causale orientée vers un but. Pour un approfondissement du style classique, cf. Bordwell (2006). The Way Hollywood Tells It, Berkeley et Los Angeles : University of California Press.

6. Zacks et Magliano (2011). Film, Narrative, and Cognitive Neuroscience. In Melcher et Bacci (dir.), Art \& the Senses, New York: Oxford University Press, pp. 435-454. Une des séquences analysées du film Moonraker est disponible en ligne : www.youtube.com/watch?v=fAucs8K5E0U

7. Le Ballon Rouge est disponible en ligne : https://www.youtube.com/watch?v=DiGFcVf34PM

8. Zacks et Magliano (2011), op. cit.

9. Cf. Bazin (1958). Montage interdit. In Qu'est-ce que le cinéma ?, 20é éd., Paris : Cerf, pp. 49-61.

10. Zacks et al. (2010), op. cit.

11. Ibid., p. 11, notre traduction: "The Red Balloon contains many long tracking shots of characters running, which may render low-level movement information less predictive ».

12. Corbetta, Patel et Shulman (2008). The Reorienting System of the Human Brain: From Environment to Theory of Mind. Neuron, 58, pp. 306-324.

13. Jacob et Jeannerod (2003). Ways of Seeing. The Scope and Limits of Visual Cognition. New York: Oxford University Press. 
14. Magliano et Zacks (2011). The Impact of Continuity Editing in Narrative Film on Event Segmentation. Cognitive Science, 35 (8), pp. 1515, notre traduction : «Intentionally breaking the rules of continuity editing may be artistically desirable and may not disrupt early perceptual processing of a film (Germeys \& d'Ydewalle, 2007). However, there may be costs for violating these conventions in terms of comprehension and memory ».

15. Zacks et al. (2011). Prediction Error Associated with the Perceptual Segmentation of Naturalistic Events. Journal of Cognitive Neuroscience, 23 (12), pp. 4057-4066.

16. Ibid., p. 4065, notre traduction : « Linking attention - and particularly memory updating - to prediction failures may be highly adaptive. If prediction failures tend to occur at those points in time at which a meaningful new event has begun, then that is just the time at which one should update one's mental models of "what is happening now" [...] This mechanism may be profoundly valuable for adaptively regulating immediate behavior as well as for guiding long-term learning about one's environment " .

17. Cf. Decety et Lamm (2007). The Role of the Right Temporoparietal Junction in Social Interaction: How Low-Level Computational Processes Contribute to Meta-Cognition. Neuroscientist, 13 (6), pp. 580-593.

18. Hasson et al. (2008). A Hierarchy of Temporal Receptive Windows in Human Cortex. Journal of Neuroscience, 28 (10), pp. 2539-2550.

19. L'extrait de City Lights, qui se trouve à 14,10 minutes du début de la vidéo, est constitué de la scène du «suicide» ou de la rencontre entre Charlot et l'homme riche : https:// www.youtube.com/watch?v=sLsapVVYM2A

20. Hasson et al. (2009). Reliability of cortical activity during natural stimulation. Trends in Cognitive Sciences, 14 (1), p. 45, notre traduction: "The order of the exact same set of events within a temporal sequence can have a strong effect on the responses in brain areas that accumulate information over time, but have little effect on areas with short TRWs. The short TRWs observed in the early visual cortex support the notion that these brain areas are optimized for rapidly processing the instantaneous visuospatial properties of a stimulus. Many cognitive processes (e.g. verbal communication, prediction, event segmentation, theory of mind, etc.), however, require accumulation of information over time ".

21. Hasson et al. (2008), op. cit. p. 2547, notre traduction : «We interpret the strong response amplitudes in these brain regions as reflecting incessant processing, presumably aimed to extract meaningful information from the stimuli. At the same time, the low reliability of the responses to temporally disrupted movies indicates a failure to attain a consistent sequence of neural states in those regions (and, correspondingly, of cognitive states) while viewing the nonecological stimuli ».

22. Schaeffer (2010). Esthétique et styles cognitifs : le cas de la poésie. Critique, $\mathrm{n}^{\circ}$ 752-753, pp. 59-70.

23. Hasson et al. (2008), op. cit., p. 2548, notre traduction : «We conjecture, therefore, that each brain area is tuned to extract information at the range of time scales that is ecologically appropriate for the content it processes. Accordingly, it should be harder to extract information at a pace that is significantly faster or slower than this ecologically determined range ».

24. Zwaan (2008). Time in Language, Situation Models, and Mental Simulations. Language Learning 58 (1), pp. 13-26.

25. Cutting et al. (2011). Quicker, Faster, Darker : Changes in Hollywood Film over 75 years. $i$ Perception, 2, pp. 569-576.

26. Fairhall et al. (2014). Temporal Integration Windows for Naturalistic Visual Sequences. PLoS ONE, 9 (7), URL: http://journals.plos.org/plosone/article?id=10.1371/journal.pone. 0102248

27. Pamilo et al. (2012). Functional Subdivision of Group-ICA Results of fMRI Data Collected during Cinema Viewing. PLoS ONE, 7 (7), URL: http://www.plosone.org/article/ 
info\%3Adoi\%2F10.1371\%2Fjournal.pone.0042000. Le film At Land (Maya Deren, 1944) est disponible en ligne : https://www.youtube.com/watch?v=0VMV0j6XVGU

28. Ibid., p. 7, notre traduction: "When the dimensionality was increased from 10 to 20 components, the superior parts of the DAN [dorsal attention network] detached from the inferior network and visual areas. The superior DAN component seemed to behave more like a top-down attention network, without coupling to the visual areas ».

29. Ibid., notre traduction: «In the 58-IC decomposition, the MPFC was split into two DMN [default-mode network] components, one covering the ventral and the other the dorsal part of the MPFC. According to previous imaging studies, the dorsal MPFC is involved in affective and emotional processing, whereas the ventral MPFC is activated during attention-demanding cognitive processing. "

30. Cf. Mars et al. (2012). On the relationship between the "default mode network" and the «social brain ». Frontiers in Human Neuroscience, 6, pp. 1-9, URL: http://journal.frontiersin.org/ Journal/10.3389/fnhum.2012.00189/full

\section{ABSTRACTS}

Event segmentation is one of the cognitive mechanisms guiding us in the comprehension of the real world and of the represented world, through the perception of the structure of these flows of events. The analysis of the main experiments on the segmentation of films, which interpret this cognitive mechanism from a reductive point of view, i.e. from a point of view exclusively referred to the process of a continuous and chronological perceptual flow, tries to show the role of segmentation in the comprehension of films through the cognitive differences relating to the diversity of the temporal structures of these narratives. From this aesthetic approach, we focus on the phenomenological aspects of these experiences, describing this processing of segmentation which drives our comprehension of these event structures across their own rhythms. Thus, we propose that one of the cognitive implications of these formal distinctions corresponds to the difference between a more pragmatic and a more subjective comprehension of the cinematographic narratives.

La segmentation des événements est un des mécanismes cognitifs qui nous guident dans la compréhension du monde réel et du monde représenté, par la perception de la structure de ces flux d'événements. À partir de l'analyse des principales expérimentations sur la segmentation des films, qui interprètent ce mécanisme cognitif d'un point de vue réductif, à savoir relatif exclusivement au traitement cognitif d'un flux perceptif continu et chronologique, nous essayons de montrer le rôle de la segmentation dans la compréhension des films grâce aux différences cognitives résultant de la diversité des structures temporelles de ces récits. Par cette approche esthétique, nous nous focalisons sur les aspects phénoménologiques de ces expériences, décrivant ce processus cognitif de segmentation qui mène à la compréhension de ces objets événementiels à travers leurs propres rythmes. Ainsi, nous proposons qu'une des implications cognitives de ces distinctions formelles correspond à la différence entre une compréhension plus pragmatique ou plus subjective des récits cinématographiques. 
INDEX

Mots-clés: esthétique, segmentation, processus cognitifs, attention, phénoménologie, montage cinématographique

Keywords: aesthetics, segmentation, cognitive process, attention, phenomenology, film editing

\section{AUTHOR}

MÁRCIA AURÉLIO BALDISSERA

CRAL/EHESS 